\title{
Transforaminal Epidural Steroid Injection - A Promising Insight in Pain Intervention
}

\author{
Misbah Salaria ${ }^{1^{*}}$, Nawaf Sheikh ${ }^{2}$ and Nandita Mehta $^{3}$ \\ ${ }^{1 *}$ Senior Resident, Department of Anesthesiology, Acharya Shri Chander College of Medical Sciences, Jammu, J\&K, India \\ ${ }^{2}$ PG Resident 3rd Year, Department of Pathology, Acharya Shri Chander College of Medical Sciences, Jammu, J\&K, India \\ ${ }^{3}$ HOD Dept of Anaesthesiology and Pain Management, Acharya Shri Chander College of Medical Sciences, Jammu, J\&K, India
}

Received: July 07, 2017; Accepted: November 15, 2017, Published: December 05, 2017

*Corresponding author: Misbah Salaria, Senior resident, Department of Anaesthesiology and Intensive Care, Acharya Shri Chander College of Medical Sciences and Hospital, Jammu. House.NO.7, C/O DR.A.Q. Salaria, Doctors Lane, Bypass Road, Narwal, Channi, Jammu, J\&K, India, 180006, Tel.: 9419134777; E-Mail Address- misbahsalaria7@gmail.com

\section{Abstract \\ Background: Back pain of lumbar origin known as "Lumbosacral Radiculopathy" is a debilitating ailment in today's modern world. The treatment modalities have been many over years ranging from prolonged bed rest, the use of analgesic medications to surgical decompression.}

Objective: In our study we have used a minimally invasive technique called the Transforaminal Epidural Steroid Injection using the steroid Triamcinolone under Fluoroscopic guidance in 30 patients of low back pain, lumbar in origin in order to alleviate pain, bridging the gap between conservative management and invasive surgical decompression with high morbidity rates.

Design: It is an observational study that we started conducting in the year 2015 after obtaining the institutional ethical committee consent in our Department of Anesthesiology and Pain Management, Acharya Shri Chander College of Medical Sciences and Hospital, Jammu. The study was a prospective randomized controlled study from 1st June, 2015 - 1st June, 2016.

Patients: 30 patients ASA class- (healthy patient or a patient with mild, well controlled systemic disease) in the age group 18- 70 years were enrolled for the study. Patients were treated with $1 \mathrm{ml}(20 \mathrm{mg})$ of Triamcinolone and $2 \mathrm{ml}$ of $0.5 \%$ Bupivacaine via transforaminal route under fluoroscopic guidance.

Outcomes Measured: VAS score, SLR measurement, drug dose intake reduction, and patient satisfaction score.

Results: Fluoroscopic guided TFESI is a modality quite promising in treating low back pain of lumbar origin for it benefit of delivering the drug in close vicinity to the target. There was a significant improvement in the VAS score, SLR measurement, drug dose intake reduction, patient satisfaction score.

Conclusion: In order to bridge the gap between conservative measures and much invasive surgical decompression, TFESI is an intervention that has shown great efficacy with regard to patient satisfaction both socially as well as physically.

Keywords: Transforaminal Epidural Steroid Injection Lumbosacral Radiculopathy; Triamcinolone;

\section{Introduction}

Lumbosacral Radiculopathy is a common medical and socioeconomic malady, with a lifetime prevalence estimated to be around 40\%-60\% (F Balague et al Lancet).

It accounts for the 2nd most common morbidity worldwide next only to dental caries [1]. The treatment modalities used over years have been many ranging from prolonged bed rest to use of analgesics, sedatives, muscle relaxants, acupuncture injections, diathermy, electrotherapy, traction and surgical decompression. Every modality of treatment comes with its own benefits and pitfalls. Epidural steroid injections are an upcoming modality that appears promising, for its benefit of delivering the drug close to the target site. The use of minimally invasive epidural corticosteroid injections for the treatment of radiculopathy was first reported in 1953. The goal of this treatment is to reduce morbidity and bridge the gap between conservative management and surgical interventions.

Most sources agree on the urgent indications for surgical intervention in patients with lumbosacral radiculopathy eg, significant/severe and progressive motor deficits, cauda equina syndrome with bowel and bladder dysfunction [2].

Caudal epidural injection was introduced in 1901 by Cathelin, Pasquier, Leri and Sicard. Cathelin injected cocaine into the epidural space. The advent of the interlaminar approach to the epidural space was considered at the time as a preferable route, as it is directed more closely to the assumed site of pathology than the caudal, thus facilitating the delivery of the drug directly to its target and also it had an additional benefit of delivering a less volume. However, subsequently, the disadvantages of the interlaminar approach, including extradural placement of the needle, which may go unrecognized without fluoroscopic guidance, the non- selectivity of the side of lumbosacral radiculopathy involved and various other disadvantages and reports of the failure of interlaminar epidural steroids to provide statistically significant improvement, raised questions not only 
about interlaminar epidural administration of steroids but also about administration of corticosteroids in itself [5].

Transforaminal lumbar epidural injections have been emerging as a superior alternative to the previously used interlaminar and caudal epidural injection techniques. Epidural steroid injections are currently used by many medical professionals for the treatment of lumbosacral radiculopathy. It was then realized that performing "blind" epidural steroid injection lacks target specificity that often results in incorrect delivery of medication to the lesion therefore, Imaging-guided steroid injections are now becoming more popular.

Lumbar transforaminal epidural steroid injection is a procedure designed to deliver an aliquot of a corticosteroid preparation mixed with a Local Anaesthetic to the immediate vicinity of a lumbar spinal nerve and its root via the intervertebral foramen in which the target nerve lies [3]. Compared with an interlaminar or caudal epidural steroid injection, a transforaminal approach provides minimal risk of dural puncture, better delivery of medication to the site of radiculopathy, lesser quqntity of drug required and increased spread into the ventral epidural space. Subsequently, only a low volume of concentrated medication necessary to produce the desired effect reduced the risk of inadvertent intrathecal injection. So now, TFESI is widely used for the management of lumbosacral radiculopathy.

Most clinicians today agree that image-guided transforaminal epidural injections are preferred to an interlaminar or caudal approach. Transforaminal ESI has been developed to administer the most target-specific agent just close to the affected nerve root. Injections are made directly into the neural foramen, which is the tip of the ear of "Scottie dog" (L Kalichman, 2008) as appears under fluoroscopic guidance just above the opening for the nerve root and outside the epidural space. It allows spread of steroid to the anterior epidural space, which is believed to be the site of discnerve interface. Recent outcome studies reveal greater duration of pain relief and avoidance of surgery compared with interlaminar injections. With the conventional TFESI technique employed, a spinal needle is positioned within the "Safe Triangle" (Sitzman BT, 2003) with the bevel below the inferior aspect of the pedicle [10]. A safe triangle is described with its sides corresponding to the horizontal base of the pedicle, the exiting nerve root and the posterolateral border of the vertebral body [6].

The transforaminal technique involves positioning of a needle in the safe triangle without pain provocation. A TFESI using a small volume of local anaesthetic will anaesthetize the spinal nerve and also partially anaesthetize the dura, the posterior longitudinal ligament, the intervertebral disc and the facet joint, all commonly involved in low back pain. But with the transforaminal approach, injection at more than one levels may be required depending upon the sites of disc herniation and radiculopathy [4].

Local Anaesthetics (LA) exert their analgesic effects by blocking the nerve conduction via their effects on $\mathrm{Na}+$ channels and suppressing the ectopic signal generation in injured nerves. In addition to providing temporary pain relief, LA provides prolonged benefits by putatively interrupting the cycle of pain.
Steroids have an anti inflammatory effect, stabilize the neuronal membranes, suppress ectopic discharge within sensitized dorsal root ganglion nerve and have a direct effect on C-fibres responsible for pain. Steroids widely used in ESI to begin with were Dexamethasone and Betamethasone, which are non- particulate steroids. Studies signify that particulate steroids though have a less favourable risk profile demonstrate nonstatistically significant superiority over non-particulate steroids.

\section{Material and Methods}

We started conducting our study in the year 2015 after obtaining the institutional ethical committee consent in our Department of Anesthesiology and Pain Management, Acharya Shri Chander College of Medical Sciences and Hospital, Jammu. The study was a retrospective randomized controlled study from 1st June, 2015 - 1st June, 2016.

30 patients ASA class- (healthy patient or a patient with mild, well controlled systemic disease) in the age group 18- 70 years were opted for the study. Patients were treated with $1 \mathrm{ml}$ (20 mg) of Triamcinolone and $2 \mathrm{ml}$ of $0.5 \%$ Bupivacaine via transforaminal route under fluoroscopic guidance.

Inclusion criteria included - History of back pain for < 1year, age 18 -70 years, patients taking analgesics such as opioids, NSAIDS or neuropathic medications such as Pregabalin, symptoms suggestive of sensory impairment in the affected limb, neurological imaging suggestive of spinal canal stenosis or herniated/degenerated disc and patients with positive SLR.

Exclusion criteria included- Patient refusal, coagulopathy, pregnancy/lactation, bleeding diathesis, infection at the site of injection, uncontrolled hypertension, uncontrolled diabetics, history of any surgery on spine, history of trauma to spine, sensitivity to the study drugs, bilateral radiculopathy, motor dysfunction involving bowel/bladder involvement and evidence of compression of Cauda Equina.

Written and informed consent was taken from all patients. Patients were asked a detailed history about duration of back pain, areas where the pain radiated, difficulty performing Activities of Daily Living (ADLs) and walking ability. Patients were explained in detail about the VAS and SLR test. A detailed account of the analgesic intake was taken from the patient. A thorough back examination was done for any visible deformity, infection at the site of injection or previous back surgery. Before being brought to the operating room, a 16-18G intravenous cannula was secured in the patients. Inj Midazolam $0.5 \mathrm{mg}$ intravenously was given prior to the procedure if required in order to alleviate anxiety.

In the operating room, routine monitors were attached (NIBP, Spo2, ECG) and then the patients were made to lie in prone position. Under all aseptic precautions, the skin overlying the target area was draped and then anesthetized with around $2 \mathrm{ml}$ of $1 \%$ Lignocaine. The level of the epidural injection was chosen depending on Magnetic Resonance Imaging (MRI) findings and physical examination of the patient. A 22- gauge spinal needle was then advanced under fluoroscopic guidance aiming the needle at the superior and anterior part of the intervertebral 
foramen. The needle placement was confirmed after injecting 1-2 ml of Isohexol, non ionic water soluble contrast medium dye, which greatly reduces the possibility of misplacement or intravascular placement of the needle, which is always a risk in epidural steroid injections performed blindly. After reaching the target, the correct needle placement was confirmed both on AP and lateral views.

After the injection, patients were repositioned and then transferred to the recovery area, where they were observed for any neurological deficit. Patients were assessed at 30 mins, 2 weeks and then 1 month after the procedure. The patients were advised not to drive and avoid any strenuous activities for the day and an attendant would accompany them that day. They were asked to report in case of any untoward incidence such as any kind of motor deficit or inability to void bladder.

In addition the patients received regular physiotherapy (short wave diathermy), were properly taught back and leg exercises and were reassessed after 2 weeks and then after 1 month.

\section{Results}

None of the patients withdrew from this study. The VAS scores were less and statistically significant in patients 30 minutes postinjection, at the 2 nd week and after 1 month. The $\mathrm{p}$ value thus being $<0.0001$, which is statistically significant. (Table 1,2 )

\begin{tabular}{|c|c|c|c|}
\hline \multicolumn{2}{|l|}{ Parameters } & \multicolumn{2}{|c|}{ Group T } \\
\hline \multicolumn{2}{|l|}{ Sex : Male: Female (n) } & \multicolumn{2}{|c|}{$40: 20$} \\
\hline \multicolumn{2}{|l|}{ Age years: mean (range) } & \multicolumn{2}{|c|}{$45.17 \pm 11.84$} \\
\hline \multicolumn{2}{|l|}{ ASA I : II (n) } & \multicolumn{2}{|c|}{$36: 24$} \\
\hline \multirow{3}{*}{\multicolumn{2}{|c|}{ Side involved }} & \multicolumn{2}{|c|}{ Right $=30$} \\
\hline & & \multicolumn{2}{|c|}{ Left $=16$} \\
\hline & & \multicolumn{2}{|c|}{ Bilateral = 14} \\
\hline \multicolumn{4}{|l|}{ Table 2: VAS Score } \\
\hline \multirow[t]{2}{*}{ Groups } & \multicolumn{3}{|c|}{ VAS Score (Mean \pm SD) } \\
\hline & 30 mins. & 2 weeks & 1 month \\
\hline Epidural Steroid Injection & $8.20 \pm 1.10$ & $\begin{array}{l}3.57 \pm \\
0.73\end{array}$ & $1.13 \pm 0.35$ \\
\hline P-value & 0.042 & $<0.0001$ & $<0.0001$ \\
\hline Remarks & $\mathrm{S}$ & $\mathrm{S}$ & $\mathrm{S}$ \\
\hline
\end{tabular}

The group comparison for mean VAS score at different time intervals is also shown in figure 1. Recovery rate of SLR test was found to be $98 \%$ with a significantly better patient satisfaction score and no pain seen in 28 out of 30 patients. $93.33 \%$ of the patients were free of pain 1 month after the commencement of TFESI treatment. (Table 3)

The drug dose intake reduction before and after the treatment was $94.33 \pm 7.28 \% 1$ month after treatment. L5 radiculopathy was the most commonly affected level, regardless of whether single or multiple levels were affected, in patients with disc prolapsed or spinal stenosis in this study. Post-ganglionic block of the L5 nerve root by L5-S1 TFESI was commonly performed.

\begin{tabular}{|l|l|l|}
\hline Table 3: Drug dose intake reduction \\
\hline \multirow{2}{*}{ Groups } & $\begin{array}{l}\text { Drug dose intake reduction (\%) } \\
\text { (Mean } \pm \text { SD) }\end{array}$ \\
\cline { 2 - 3 } & 2 weeks & 1 month \\
\hline Epidural Steroid Injection & $54.67 \pm 9.09$ & $94.33 \pm 7.28$ \\
\hline P-value & $<0.0001$ & $<0.0001$ \\
\hline Remarks & $\mathrm{S}$ & $\mathrm{S}$ \\
\hline
\end{tabular}

\section{Discussion}

Spinal Transforaminal Epidural Steroid Injections have been used for chronic pain management for over years. Major criticism of most of the early studies done on epidural steroid injection efficacy is their use of "blind" approaches and therefore, missing the target specificity. So even with skilled hands, blind epidural injections result in incorrect placement of the drug [9]. The newer minimally invasive modalities with fluoroscopy and more precise techniques of delivery such as the TFESI have recently been added to the list of available treatment options for radiculopathies for they are more likely to place medication near to the exact target site, yielding diagnostic feedback and maximizing the therapeutic effects.. (Table 4)

\begin{tabular}{|l|l|l|}
\hline Table 4: Patients satisfaction \\
\hline Patients Satisfaction & 2 weeks & 1 month \\
\cline { 2 - 3 } & $\begin{array}{l}\text { Epidural Steroid } \\
\text { Injection }\end{array}$ & $\begin{array}{l}\text { Epidural Steroid } \\
\text { Injection }\end{array}$ \\
\hline No pain & $5(16.67)$ & $28(93.33)$ \\
\hline Much improved & $20(66.67)$ & $2(6.67)$ \\
\hline Slightly improved & $5(16.67)$ & $0(0.00)$ \\
\hline No effects & $0(0.00)$ & $0(0.00)$ \\
\hline P-value & $<0.0001$ & \\
\hline Remarks & $\mathrm{S}$ & \\
\hline
\end{tabular}

This study consisted of 30 patients treated with transforaminal epidural steroid injection with a history of persistent low back pain with radiculopathy secondary to disc herniation. Patients showed statistically significant improvement in all parameters. At the end of 1 month, significant improvement was seen in 93\% of patients. Overall relief achieved in view of patient satisfaction was $94.33 \pm 7.28$ of drug dose intake reduction over a period of 1 month with 3 procedures when TFESI was used. According to the VAS score, $1.13 \pm 0.35$ improvement was seen, which showed statistical significance. Thus, this study provides evidence that in carefully selected patients, with repeat injections, patients respond well to TFESI with a much higher level of patient satisfaction score and greater reduction in drug dose intake in the ESI group.

Lumbar radiculopathy can be well managed conservatively though, but many patients still have persistent disabling radicular pain demanding great attention in a pain specialty clinic. The threshold of offering TFESI as a diagnostic tool or a therapeutic adjunct to conservative treatment is variable among pain specialists, reflected by the wide variation of symptom chronicity 
from days to years among the 30 patients receiving TFESI in this study. Other factors affecting the threshold of offering TFESI include lumbar radiculopathy not controlled by more than three different kinds of analgesic combinations in a high dose acting on different pain pathways, and the diagnostic need for clinical MRI correlations, especially among patients contemplating surgerical decompression

\section{Conclusion}

Lumbar Disc Herniation (LDH) is a common pathology. Fortunately, most patients with lower extremity radiculitis and/ or radiculopathy will note symptom resolution without surgical intervention(s), whereas most will require surgical intervention.

An often used adjunct to nonsurgical treatment is Transforaminal Epidural Steroid Injection (TFESI) [7]. Focused placement of steroid medication at the injured nerve root addresses the specific pathophysiologic changes incurred owing to the disc herniation rendering great benefit with pain relief Several studies have demonstrated the benefit of TFESI in treating radicular symptoms secondary to LDH and the success of TFESI compared with other injection techniques has also been demonstrated.

Relatively less severe pathologies, such as disc herniations, are further delayed to ensure timely management of more serious or emergent conditions introducing harm to the patient, as symptoms become chronic and more resistant to manage. Chronicity affects mood, fatigue, employability and social functioning leading to greater morbidity.This environment demands an effort to find alternate treatment techniques to alleviate the need for surgical interventions. Successful nonsurgical treatment reduces surgical wait times and costs to an overburdened health care system [8]. Transforaminal epidural steroid injection has proven to be an effective early intervention for radiculopathy secondary to LDH, but its effectiveness is unproven in patients demonstrating more longstanding symptoms.

\section{References}

1. Alan P. Winnie, J. Theodore Hartman, H-Lee Meyers, et al. Intradural and extra dural corticosteroid injections in the management of low back ache. Anaesth Analg. 1972;51(6):990-999.

2. Balagué F, Mannion AF, Pellisé F, Cedraschi C. Non-specific low back pain. Lancet. 2012;379(9814):482-491. doi: 10.1016/S01406736(11)60610-7

3. Benyamin RM, Manchikanti L, Parr AT, Diwan S, Singh V, Falco FJ, et al. The effectiveness of lumbar interlaminar epidural injections in managing chronic low back and lower extremity pain. Pain Physician. 2012;15:363-404.

4. Bicket MC, Gupta A, Brown $\mathrm{CH} 4$ th, Cohen SP. Epidural injections for spinal pain: A systematic review and meta-analysis evaluating the "control" injections in randomized controlled trials. Anesthesiology. 2013;119(4):907-931. doi: 10.1097/ALN.0b013e31829c2ddd

5. Blankenbaker DG, Davis KW, Choi JJ. Selective nerve root blocks. Semin Roentgenol. 2004;39:24-36.

6. Botwin KP, Gruber RD, Bouchlas CG, Torres-Ramos FM, Sanelli JT, Freeman ED, et al. Fluoroscopically guided lumbar transforaminal epidural steroid injections in degenerative lumbar stenosis. Am J Phys Med Rehabil. 2002;81:898-905.

7. Cyriax JH. Cervical Disc Herniation. Textbook of Orthopaedic Medicine, 3rd edition. London: Cassell. 1957: 460-469.

8. Nelson DA, Landau WM. Intraspinal steroids: history, efficacy, accidentality, and controversy with review of United States Food and Drug Administration reports. Neurol Neurosurg Psychiatry. 2001;70:433-443.

9. E. N. Coomes. Comparison between epidural anaesthesia and bed rest in management of sciatica. British Medical Journal 7. 1961;20-24.

10.el-Khoury GY, Ehara S, Weinstein JN, Montgomery WJ, Kathol MH. Epidural steroid injection: a procedure ideally performed with fluoroscopic control. Radiology. 1988;168:554-557. 